\title{
МЕТОД ДИСКРЕТНЫХ ОРДИНАТ ПРИМЕНИТЕЛЬНО К РЕШЕНИЮ УРАВНЕНИЯ ПЕРЕНОСА ИЗЛУЧЕНИЯ В ОДНОРОДНОЙ ПЛОСКОПАРАЛЛЕЛЬНОЙ АНИЗОТРОПНО РАССЕИВАЮЩЕЙ АТМОСФЕРЕ. 2
}

(Представил Г. Кузмин)

Одним из возможных методов решения интегро-дифференциального уравнения переноса является метод дискретных ординат. В [ $\left.{ }^{1}\right]$ был рассмотрен один из вариантов этого метода: интегро-дифференциальное уравнение приближенно заменяется системой линейных дифференциальных уравнений с краевыми условиями, которая получается путем замены интегрального члена некоторой квадратурной суммой. Было показано, что при «равномерной» дискретизации уравнения переноса по азимуту матрица коэффициентов системы дифференциальных уравнений разбивается на блоки - циркулянты, которые легко приводятся с помощью унитарного преобразования, не зависящего от конкретного блока, к диагональному виду. Әто позволило разбить приближенную задачу большой размерности на подзадачи меньшей размерности.

В настоящей работе исследуется класс систем дифференциальных уравнений с краевыми условиями, который интерпретируется как класс приближенных методов решения уравнения переноса. Сюда входят, например, дискретные методы решения уравнения переноса излучения, рассмотренные в $\left[{ }^{1-4}\right]$. Для решения системы из этого класса предлагается итерационный метод Зейделя. Устанавливается сходимость и оценивается скорость сходимости этого метода (теорема 1). Для решения задачи Коши, которая возникает на каждом итерационном шаге, обсуждается метод трапеций с переменным шагом. Показано, что при определенных ограничениях на шаг решение этой задачи положительно (теорема 2). В двух последних разделах приводятся два приближенных метода решения уравнения переноса излучения из рассматриваемого класса, использующие «равномерную» дискретизацию по азимуту, и их численные реализации на конкретном примере.

\section{1. Постановка задачи}

Рассмотрим одну из основных краевых задач атмосферной оптики

$$
\begin{gathered}
\mu \frac{d J(\tau, \mu, \varphi)}{d \tau}=-J(\tau, \mu, \varphi)+\lambda(4 \pi)^{-1} \int_{0}^{2 \pi} \int_{-1}^{+1} g\left(\gamma^{\prime}\right) J\left(\tau, \mu^{\prime}, \varphi^{\prime}\right) d \mu^{\prime} d \varphi^{\prime}+ \\
+F g(\gamma) \exp (\sigma \tau), \quad 0 \leqslant \tau \leqslant H, \quad-1 \leqslant \mu \leqslant 1, \quad 0 \leqslant \varphi \leqslant 2 \pi, \\
J(0, \mu, \varphi)=0 \text { при } \mu>0, \jmath(H, \mu, \varphi)=0 \text { при } \mu<0 .
\end{gathered}
$$

Задача (1)-(2) описывает перенос излучения в освещенной параллельными лучами анизотропно рассеивающей плоскопараллельной сре- 
де оптической толщины $H$ и вероятностью $\lambda(0 \leqslant \lambda \leqslant 1)$ выжива̀нйя кванта в акте столкновения с частицей среды. Анизотропность рассеяния учитывается индикатрисой рассеяния $g(\gamma) \geqslant 0$, нормированной условием

$$
\begin{gathered}
(4 \pi)^{-1} \int_{\omega} g\left(\gamma^{\prime}\right) d \omega=(4 \pi)^{-1} \int_{-1}^{+1} \int_{0}^{2 \pi} g\left(\gamma^{\prime}\right) d \mu^{\prime} d \varphi^{\prime}=1, \\
\cos \gamma^{\prime}=\mu \mu^{\prime}+\sqrt{\left(1-\mu^{2}\right)\left(1-\mu^{\prime 2}\right)} \cos \left(\varphi-\varphi^{\prime}\right), \\
\cos \gamma=\mu \mu_{0}+\sqrt{\left(1-\mu^{2}\right)\left(1-\mu_{0}^{2}\right)} \cos \left(\varphi-\varphi_{0}\right), \quad \gamma, \gamma^{\prime} \in[0, \pi] .
\end{gathered}
$$

Здесь (- $\left.\arccos \mu_{0}, \varphi_{0}\right)$ - направление прямого излучения в сферической системе координат с полярной осью, идущей в сторону растущих оптических глубин, $\sigma=-1 / \mu_{0}$. Искомая функция $\jmath(\tau, \mu, \varphi)$ это интенсивность излучения на оптической глубине $\tau$ в направлении, которое определяется полярным расстоянием $\arccos \mu$ и азимутом $\varphi$.

Для решения задачи (1)-(2) рассмотрим следующий класс дискретных методов

$$
\begin{gathered}
a_{s m} \frac{d J_{s m}(\tau)}{d \tau}=-b_{s m} J_{s m}(\tau)+\lambda(4 \pi)^{-1}\left[\sum_{j=1}^{n} \sum_{l=1}^{p} g_{j l s m} J_{j l}(\tau)+\right. \\
\left.+\sum_{j=1}^{n} \sum_{l=1}^{p} g_{-j l s m} J_{-j l}(\tau)\right]+u_{s m} \exp (\sigma \tau), \\
J_{i m}(0)=J_{-i m}(H)=0, \quad s= \pm 1, \pm 2, \ldots, \pm n ; \quad i=1,2, \ldots, n, \\
m=1,2, \ldots, p .
\end{gathered}
$$

На параметры $a_{s m}, b_{s m}$ и $g_{j l s m}$ наложим следующие ограничения:

$$
\begin{gathered}
a_{i m}>0, b_{i m}>0, a_{-i m}=-a_{i m}, b_{-i m}=b_{i m}, \\
g_{i l j m} \geqslant 0, \\
g_{i l j m}=g_{-i l(-j) m}, g_{i l(-j) m}=g_{-i l j m}, g_{i l j m}=g_{j l i m}=g_{j m i l}, \\
b_{i m}^{-1} \sum_{j=1}^{n} \sum_{l=1}^{p}\left(g_{j l i m}+g_{-j l i m}\right)=4 \pi, \\
i, j=1,2, \ldots, n ; \quad m, l=1,2, \ldots, p .
\end{gathered}
$$

Отметим, что свойство (9) является дискретным аналогом условия (3). Сформулированная нами задача имеет единственное решение. Для доказательства этого факта достаточно дословно повторить рассуждения раздела 2 работы $\left[{ }^{1}\right]$.

\section{2. Векторно-матричная запись системы (4)-(5)}

Используя векторно-матричную символику и учитывая свойства и (8), перепишем задачу (4)-(5) в виде

$$
\begin{gathered}
A_{i} \frac{d J_{i}(\tau)}{d \tau}=-B_{i} \Im_{i}(\tau)+\sum_{j=1}^{n}\left(G_{i j} \Im_{j}(\tau)+G_{-i j} \jmath_{-j}(\tau)\right)+u_{i} \exp (\sigma \tau), \\
A_{i} \frac{d \jmath_{-i}(\tau)}{d \tau}=B_{i} J_{-i}(\tau)-\sum_{j=1}^{n}\left(G_{-i j} \jmath_{j}(\tau)+G_{i j} J_{-j}(\tau)\right)-u_{-i} \exp (\sigma \tau), \\
J_{i}(0)=J_{-i}(H)=0, \quad i=1,2, \ldots, n
\end{gathered}
$$


Здесьь

$$
\begin{aligned}
& \Im_{i}(\tau)=\left(\Im_{i 1}(\tau) \Im_{i 2}(\tau) \ldots \Im_{i p}(\tau)\right)^{\mathrm{T}}, \\
& u_{i}=\left(\begin{array}{llll}
u_{i 1} & u_{i 2} & \ldots & u_{i p}
\end{array}\right)^{\mathrm{T}} \text {, }
\end{aligned}
$$

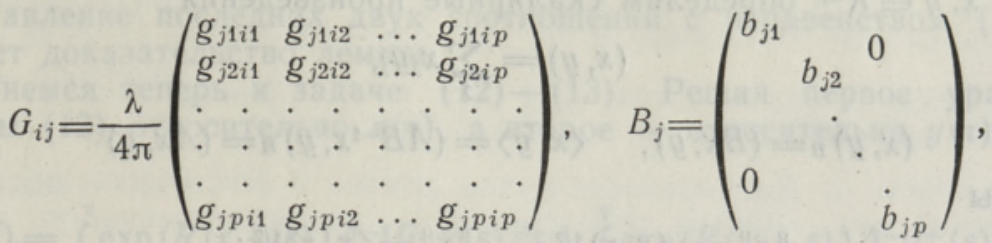

$$
\begin{aligned}
& A_{j}=\left(\begin{array}{ccc}
a_{j 1} & & \\
& a_{j 2} & \\
& \cdot & \\
0 & & \cdot \\
& & a_{j p}
\end{array}\right), \quad i= \pm 1, \pm 2, \ldots, \pm n ; \quad j=1,2, \ldots, n .
\end{aligned}
$$

Кроме этой, будем использовать еще одну форму записи задачи $(4)-(5)$

$$
\begin{gathered}
x^{\prime}(\tau)=R x(\tau)+A^{-1} G^{-} y(\tau)+A^{-1} f^{+}(\tau), \\
y^{\prime}(\tau)=-R y(\tau)-A^{-1} G-x(\tau)-A^{-1} f^{-}(\tau), \\
x(0)=y(H)=0 .
\end{gathered}
$$

Здесь $R=A^{-1} B\left(B^{-1} G^{+}-I\right)$,

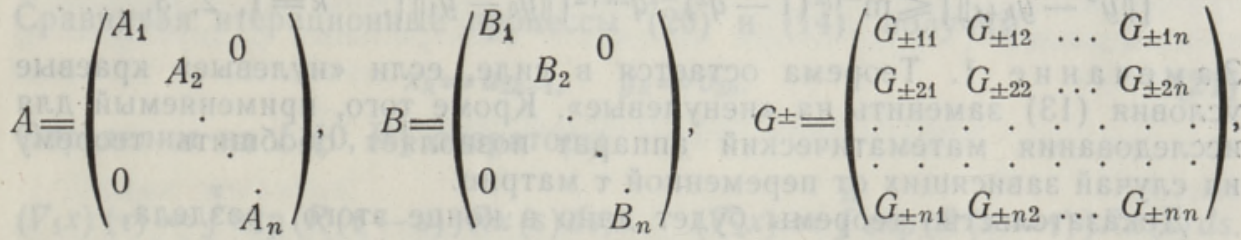

$$
\begin{aligned}
& x(\tau)=\left(\mathfrak{J}_{1}(\tau) \mathfrak{J}_{2}(\tau) \ldots J_{n}(\tau)\right)^{\mathrm{T}}, y(\tau)=\left(\mathfrak{J}_{-1}(\tau) \mathfrak{J}_{-2}(\tau) \ldots J_{-n}(\tau)\right)^{\mathrm{T}}, \\
& f^{ \pm}=\exp (\sigma \tau)\left(u_{ \pm 1} u_{ \pm 2} \ldots u_{ \pm n}\right)^{\mathrm{T}} \text {, }
\end{aligned}
$$

а $I$ - единичная матрица.

\section{3. Итерационный процесс}

Рассмотрим итерационный процесс

$$
\begin{aligned}
& x_{k+1}^{\prime}(\tau)=R x_{k+1}(\tau)+A^{-1} G^{-} y_{k}(\tau)+A^{-1} f^{+}(\tau), \\
& x_{k+1}(0)=0, \\
& y_{k+1}^{\prime}(\tau)=-R y_{k+1}(\tau)-A^{-1} G^{-} x_{k+1}(\tau)-A^{-1} f^{-}(\tau), \\
& y_{k+1}(H)=0 .
\end{aligned}
$$

Для описания этого итерационного процесса введем ряд обозначений и определений, а именно:

1) $g=\min _{\substack{1 \leqslant s \leqslant n \\ 1 \leqslant m \leqslant p}}\left(4 \pi b_{s m}\right)^{-1} \sum_{j=1}^{n} \sum_{l=1}^{p} g_{j l s m} ; \quad G=\max _{\substack{1 \leqslant s \leqslant n \\ 1 \leqslant m \leqslant p}}\left(4 \pi b_{s m}\right)^{-1} \sum_{j=1}^{n} \sum_{l=1}^{p} g_{j l s m}$; 
2) $\mathrm{m}=(1-\lambda \bar{G}) \min _{\substack{1 \leqslant i \leqslant n \\ 1 \leqslant m \leqslant p}} a_{i m}^{-1} b_{i m} ; \quad M=(1+\lambda G) \max _{\substack{1 \leqslant i \leqslant n \\ 1 \leqslant m \leqslant p}} a_{i m}^{-1} b_{i m}$;

3) для $x, y \in R$ пр определим скалярные произведения

$$
(x, y)=\sum_{j} x_{j} y_{j}
$$

$$
(x, y)_{B}=(B x, y), \quad\langle x, y\rangle=\left(A B^{-1} x, y\right)_{B}=(A x, y)
$$

и нормы

$$
\|x\|_{B}=(x, x)_{B}^{1 / 2}, \quad\|x\|=\langle x, x\rangle^{1 / 2} ;
$$

4) $L_{2}[0, H]-$ пространство вектор-функций $x(\tau)$, определенных на отрезке $[0, H]$ и принимающих значения в $R$ пр, в котором норма задается как

$$
\|\| x \| \mid=\left(\int_{0}^{H}\|x\|^{2}(\tau) d \tau\right)^{1 / 2}
$$

5) $\theta_{1}=\lambda(1-g)(1-\lambda g)^{-1}$;

6) $\theta_{2}=1-\exp (-M H)$;

7) $q=\theta_{1} \theta_{2}$.

Т еорем а 1. Нтерационный процесс (14) сходится при любом начальном приближении $y_{0} \in L_{2}[0, H]$. Нмеют место оценки

$$
\begin{aligned}
& || x^{*}-x_{k+1}\left\|\left|\leqslant \mathrm{~m}^{-1 / 2}\left(1-q^{2}\right)^{-1} q^{2 k+1}\right|\right\| y_{0}-y_{1} \| \mid, \\
& \left|\left\|y^{*}-y_{k+1}\right\|\right| \leqslant \mathrm{m}^{-1 / 2}\left(1-q^{2}\right)^{-1} q^{2 k+2}\left|\left\|y_{0}-y_{1}\right\|\right|, \quad k=1,2,3, \ldots .
\end{aligned}
$$

3 а мечание 1. Теорема остается в силе, если «нулевые» краевые условия (13) заменить на «ненулевые». Кроме того, применяемый для исследования математический аппарат позволяет обобщить теорему на случай зависящих от переменной $\tau$ матриц.

Доказательство теоремы будет дано в конце этого раздела.

Лемм а 1. Матрица $R=A^{-1} B\left(B^{-1} G^{+}-I\right)$ является самосопряженным оператором в гильбертовом пространстве со скалярным произведением $\langle$.,. $>$. Нмеет место неравенство

$$
-M\langle x, x\rangle \leqslant\langle R x, x\rangle \leqslant-\mathrm{m}\langle x, x\rangle .
$$

Доказательство. Определение матриц $A, B$ и $G^{+}$с использованием условий (8)порождает цепочку равенств

$$
\begin{aligned}
\langle R x, y\rangle & =\left(\left(B^{-1} G^{+}-I\right) x, y\right)_{B}=\left(x, G^{+} y\right)-(x, B y)= \\
& =\left(x,\left(B^{-1} G^{+}-I\right) y\right)_{B}=\langle x, R y\rangle,
\end{aligned}
$$

доказывающую самосопряженность оператора $R$.

Используя неравенство Коши-Буняковского, имеем

$$
\begin{gathered}
-\left(\left\|B^{-1} G^{+}\right\|_{B}+1\right)(x, x)_{B} \leqslant\langle R x, x\rangle=\left(B^{-1} G^{+} x, x\right)_{B}- \\
-(x, x)_{B} \leqslant-\left(1-\left\|B^{-1} G^{+}\right\|_{B}\right)(x, x)_{B} .
\end{gathered}
$$

Учитывая самосопряженность оператора $B^{-1} G^{+}$, действующего в гильбертовом пространстве со скалярным произведением $(., .)_{B}$, и определение величины $G$, получим

$$
\left\|B^{-1} G^{+}\right\|_{B} \leqslant G .
$$

Ввиду того, что матрица $A^{-1} B$ диагональна, имеет место соотношение 


$$
\left(\min _{\substack{1 \leqslant i \leqslant n \\ 1 \leqslant m \leqslant p}} a_{i m}^{-1} b_{i m}\right)\langle x, x\rangle \leqslant\left\langle A^{-1} B x, x\right\rangle=(x, x)_{B} \leqslant\left(\max _{\substack{1 \leqslant i \leqslant n \\ 1 \leqslant m \leqslant p}} a_{i m}^{-1} b_{i m}\right)\langle x, x\rangle .
$$

Сопоставление последних двух соотношений с неравенством (16) завершает доказательство леммы.

Вернемся теперь к задаче $(12)-(13)$. Решая первое уравнение системы (12) относительно $x(\tau)$, а второе - относительно $y(\tau)$, получим

$$
\begin{aligned}
& x(\tau)=\int_{0}^{\tau} \exp (R(\tau-s)) A^{-1} G^{-} y(s) d s+\int_{0}^{\tau} \exp (R(\tau-s)) A^{-1} f^{+}(s) d s, \\
& y(\tau)=\int_{\tau}^{H} \exp (R(s-\tau)) A^{-1} G^{-} x(s) d s+\int_{\tau}^{H} \exp (R(s-\tau)) A^{-1} f^{-}(s) d s
\end{aligned}
$$

или в операторном виде

$$
\begin{aligned}
& x=V_{1} y+f_{1}, \quad f_{1}(\tau)=\int_{0}^{\tau} \exp (R(\tau-s)) A^{-1} f^{+}(s) d s, \\
& y=V_{2} x+f_{2}, \quad f_{2}(\tau)=\int_{\tau}^{H} \exp (R(s-\tau)) A^{-1} f^{-}(s) d s .
\end{aligned}
$$

Рассмотрим итерационный процесс

$$
\begin{aligned}
& u_{k}=V_{1} v_{k-1}+f_{1}, \\
& v_{k}=V_{2} v_{k-1}+f_{2}, \quad k=1,2,3, \ldots .
\end{aligned}
$$

Сравнивая итерационные процессы (20) и (14), получим

$$
x_{k}=u_{2 k-1}, \quad y_{k}=v_{2 k} .
$$

Определим на $L_{2}[0, H]$ операторы

$$
\left(\widetilde{\nabla}_{1} x\right)(\tau)=\int_{0}^{\tau} \exp (R(\tau-s)) R x(s) d s, \quad\left(\nabla_{2} x\right)=\int_{\tau}^{H} \exp (R(s-\tau)) R x(s) d s .
$$

Л ем м 2. Имеют место неравенства

$$
\left|\left\|\tilde{\nabla}_{i}\right\|\right| \leqslant 1-\exp (-M H), \quad i=1,2 .
$$

Доказательство. Так как матрица $-R$ является самосопряженным положительно определенным оператором в гильбертовом пространстве со скалярным произведением $<.,$.$\rangle , то существует орто-$ нормированный базис $e_{1}, e_{2}, \ldots, e_{N}(N=n p) \quad\left[{ }^{5}\right]$, состоящий из собственных векторов оператора $-R$, соответствующих собственным числам $\mathrm{m}_{\mathrm{i}} \leqslant \gamma_{1} \leqslant \gamma_{2} \leqslant \ldots \leqslant \gamma_{N} \leqslant M$. Пусть $u \in L_{2}[0, H]$. Тогда

$$
u(\tau)=\sum_{k=1}^{N} u_{k}(\tau) e_{k}
$$

Отсюда

$$
\left\|\left(\widetilde{\nabla}_{1} u\right)(\tau)\right\|^{2}=\sum_{k=1}^{N}\left(\int_{0}^{\tau} \gamma_{k} \exp \left(-\gamma_{k}(\tau-s)\right) u_{k}(s) d s\right)^{2} .
$$

Далее

$$
\|\| \widetilde{\nabla}_{1} x \|\left.\right|^{2}=\sum_{k=1}^{N} \int_{0}^{H}\left(\int_{0}^{\tau} \gamma_{k} \exp \left(-\gamma_{k}(\tau-s)\right) x_{k}(s) d s\right)^{2} d \tau \leqslant
$$

$$
\leqslant \sum_{k=1}^{N} \int_{0}^{H}\left(\int_{0}^{\tau} \gamma_{k} \exp \left(-\gamma_{k}(\tau-s)\right) d s\right)\left(\int_{0}^{\tau} \gamma_{k} \exp \left(-\gamma_{k}(\tau-s)\right) x_{k}^{2}(s) d s\right) d \tau \leqslant
$$




$$
\begin{aligned}
& \quad \leqslant(1-\exp (-M H)) \sum_{k=1}^{N} \int_{0}^{H} x_{k}^{2}(s)\left(1-\exp \left(-(H-s) \gamma_{k}\right) d s \leqslant\right. \\
& \leqslant(1-\exp (-M H))^{2} \sum_{k=1}^{N} \int_{0}^{H} x_{k}^{2}(s) d s=(1-\exp (-M H))^{2}|\|x\||^{2} .
\end{aligned}
$$

Отсюда следует неравенство (22) для $i=1$. Доказательство леммы для $i=2$ аналогично.

3 а мечани е. 2. Для доказательства леммы 2 фактически потребовалось только два свойства оператора $R$ - самосопряженность и отрицательная определенность, поэтому в определении 3 ) норму $\|\cdot\|$ можно заменить любой другой нормой $\|\cdot\| *=(.,.) *^{1 / 2}$, лишь бы в новом пространстве оператор $R$ был самосопряженным и отрицательно определенным.

Лемм а 3. Имеют место неравенства

$$
\left|\left\|V_{i}\right\|\right| \leqslant(1-\exp (-M H))\left\|\left(B^{-1} G^{+}-I\right)^{-1} B^{-1} G^{-}\right\|, \quad i=1,2 .
$$

Док а з а тельство леммы очевидно.

Лемм а 4. Спектральный радиус е оператора $\left(I-B^{-1} G^{+}\right)^{-1} B^{-1} G^{-}$ удовлетворяет неравенству $\mathrm{\leqslant} \leqslant \theta_{1}$.

Доказ а тельство.Положим

$$
K_{1}=I-\left(B^{-1} G^{+}+t B^{-1} G^{-}\right), \quad K_{2}=t B^{-1} G^{-}, \quad K=K_{1}+K_{2}+K_{2}^{*},
$$

где $t$ - некоторый параметр. Матрицы $K, K_{1}$ и $K_{2}$ являются самосопряженными операторами в гильбертовом пространстве со скалярным произведением $(., .)_{\text {в }}$. Будем оценивать спектральный радиус $\varrho_{1}=t_{2}$ оператора

$$
C=\left(K_{1}+K_{2}\right)^{-1} K_{2}^{*}=t\left(I-B^{-1} G^{+}\right)^{-1} B^{-1} G^{-} .
$$

Имеем

$$
\begin{gathered}
(K x, x)_{B}=(x, x)_{B}-\left(\left(B^{-1} G^{+}-t B^{-1} G^{-}\right) x, x\right) \geqslant \\
\geqslant\left(1-\left\|B^{-1} G^{+}-t B^{-1} G^{-}\right\|_{B}\right)(x, x)_{B} .
\end{gathered}
$$

Так как матрицы $B^{-1} G^{+}$и $B^{-1} G^{-}$являются самосопряженными операторами в гильбертовом пространстве со скалярным произведением $(., .)_{B}$, то величина $\left\|B^{-1} G^{+}-t B^{-1} G^{-}\right\|_{B}$ есть наибольшее по абсолютной величине собственное значение матрицы $\left(B^{-1} G^{+}-t B^{-1} G^{-}\right)$. Это собственное значение с учетом (7) можно оценить следующим образом

$$
\begin{aligned}
\left\|B^{-1} G^{+}-t B^{-1} G^{-}\right\|_{B} & \leqslant \max _{\substack{1 \leqslant s \leqslant n \\
1 \leqslant m \leqslant p}} \lambda\left(4 \pi b_{s m}\right)^{-1} \sum_{j=1}^{n} \sum_{l=1}^{p}\left|g_{j l s m}-t g_{-j l s m}\right| \leqslant \\
& \leqslant \lambda \max _{\substack{1 \leqslant s \leqslant n \\
1 \leqslant m \leqslant p}}\left(\alpha_{s m}+t \beta_{s m}\right)
\end{aligned}
$$

где

Отсюда

$$
\begin{gathered}
\alpha_{s m}=\left(4 \pi b_{s m}\right)^{-1} \sum_{j=1}^{n} \sum_{l=1}^{p} g_{j l s m}, \quad \beta_{s m}=\left(4 \pi b_{s m}\right)^{-1} \sum_{j=1}^{n} \sum_{l=1}^{p} g_{-j l s m}, \\
\alpha_{s m}+\beta_{s m}=1 .
\end{gathered}
$$

$$
\left\|B^{-1} G^{+}-t B^{-1} G^{-}\right\|_{B} \leqslant \lambda \max _{\substack{1 \leqslant s \leqslant n \\ 1 \leqslant m \leqslant p}}\left((1-t) \alpha_{s m}+t\right)=\lambda((1-t) \alpha+t)=\gamma(t) .
$$


Здесь

$$
\alpha=\left\{\begin{array}{l}
G, t \leqslant 1 \\
g, t>1
\end{array}\right.
$$

Пусть $t \in\left(0,(1-\lambda g)(\lambda(1-g))^{-1}\right)$. Тогда, учитывая последнее неравенство, получим

$$
(K x, x)_{B} \geqslant(1-\gamma(t))(x, x)_{B}, \quad 1-\gamma(t)>0 .
$$

Аналогично

$$
\left(K_{1} x, x\right)_{B} \geqslant(1-\gamma(t))(x, x)_{B} .
$$

Используя теорему $\left[{ }^{6}\right]$ (c. 105) об оценке спектрального радиуса, получим

$$
Q_{1} \leqslant 1-\gamma_{0}\left\|K_{1}+K_{2}\right\|_{B}^{-1},
$$

где $\gamma_{0}$ удовлетворяет неравенству

$$
\gamma_{0} \geqslant \beta_{0}-\sqrt{\beta_{0}^{2}-(1-\gamma(t))^{2}}, \quad \beta_{0}=\sup _{\|x\|_{B}=1}\left|\left(\left(K_{1}+K_{2}\right) x, x\right)_{B}\right| .
$$

Учитывая самосопряженность оператора $K_{1}+K_{2}$ и соотношение $\varrho_{1}=t \varrho$, получим

$\mathrm{Q}^{2} \leqslant t^{-2}\left(1-(1-\gamma(t))^{2} /(1+\gamma(0))^{2}\right)=\psi(t), \quad \forall t \in\left(0,(1-\lambda g)(\lambda(1-g))^{-1}\right)$.

Нетрудно заметить, что функция $\psi(t)$ монотонно убывает. Следовательно,

$$
\varrho^{2} \leqslant \psi\left(t^{*}\right)<\psi(t), \quad t^{*}=(1-\lambda g)(\lambda(1-g))^{-1}
$$

или, что то же самое,

$$
\varrho \leqslant \lambda(1-g)(1-\lambda g)^{-1}=\theta_{1} .
$$

Лемма доказана.

Доказательство те оремы. Положим

$$
(x, y) *=\left(\left(I-B^{-1} G^{+}\right) x, y\right)_{B}=-\langle R x, y\rangle, \quad\|x\|_{*}=(x, x)_{*}^{1 / 2} .
$$

Определим в $L_{2}[0, H]$ эквивалентную норму

$$
\|\| x\left\|_{{ }^{2}}^{2}=\int_{0}^{H}\right\| x(\tau)\left\|_{*}^{2} d \tau, \quad \mathrm{m}^{1 / 2}|\|x\|| \leqslant|\|x\|| * \leqslant M^{1 / 2}|\|x\|| .\right.
$$

Очевидно, матрицы $R$ и $\left(I-B^{-1} G^{+}\right)^{-1} B^{-1} G^{+}$являются самосопряженными операторами в гильбертовом пространстве со скалярным произведением $(., \ldots)$. Отсюда следует равенство

$$
\left\|\left(B^{-1} G^{+}-I\right)^{-1} B^{-1} G^{+}\right\| *=0,
$$

где $\mathrm{Q}-$ спектральный радиус матрицы $\left(B^{-1} G^{+}-I\right) B^{-1} G^{+}$.

Обозначим $X=L_{2}[0, H] \times L_{2}[0, H]$. Введем в $X$ конусную норму $\left(\left[{ }^{6}\right]\right.$, c. 93$)$

$$
\mathrm{V} z=(x, y)^{\mathrm{T}} \in X:|z|^{*}=(|\|x\|| \cdot, \quad|\|y\|| \cdot)^{\mathrm{T}} \in R^{2}:
$$

Запишем систему (16) в операторном виде

$$
z=V z+f, \quad V: X \rightarrow X,
$$

а итерационный процесс $(20)-$ в виде

$$
z_{k+1}=V z_{k}+f, \quad z_{k}=\left(u_{k}, v_{k}\right)^{\mathrm{T}} .
$$


Пусть $z^{*}=\left(x^{*}, y^{*}\right)^{\mathrm{T}}$ - решение уравнения (23). Из лемм $2-4$ и замечания 2 следует справедливость следующих преобразований:

$$
\begin{gathered}
\left|z^{*}-z_{2 k+1}\right|^{*}=\left|V^{2} z^{*}-V^{2} z_{2 k-1}\right|^{*} \leqslant q^{2}\left|z^{*}-z_{2 k-1}\right|^{*} \leqslant \\
\leqslant q^{2}\left(\left|z^{*}-z_{2 k+1}\right|^{*}+\left|z_{2 k+1}-z_{2 k-1}\right|^{*}\right) .
\end{gathered}
$$

Учитывая, что $q<1$, получим

$$
\begin{gathered}
\left|z^{*}-z_{2 k+1}\right|^{*} \leqslant q^{2}\left(1-q^{2}\right)^{-1}\left|z_{2 k+1}-z_{2 k-1}\right|^{*} \leqslant \\
\leqslant q^{2}\left(1-q^{2}\right)^{-1} q^{2 k-1} E^{2 k-1}\left|z_{2}-z_{0}\right|^{*},
\end{gathered}
$$

где

$$
E=\left(\begin{array}{ll}
0 & 1 \\
1 & 0
\end{array}\right)
$$

Аналогично

$$
\left|z^{*}-z_{2 k}\right|^{*} \leqslant q^{2}\left(1-q^{2}\right)^{-1} q^{2 k-2}\left|z_{2}-z_{0}\right|^{*} .
$$

Учитывая (21) и эквивалентность норм $|\|\cdot\||$ и $|\|\cdot\|| *$, убеждаемся, что неравенство (24) для первой координаты дает

$$
\left|\left\|x^{*}-u_{2 k+1}\right\|\right|=\left|\left\|x^{*}-x_{k+1}\right\|\right| \leqslant m^{-1 / 2}\left(1-q^{2}\right)^{-1} q^{2 k+1}\left|\left\|y_{0}-y_{1}\right\|\right|,
$$

а неравенство (25) для второй координаты дает

$$
\left|\left\|y^{*}-y_{k+1}\right\|\right| \leqslant m^{-1 / 2}\left(1-q^{2}\right)^{-1} q^{2 k+2}\left|\left\|y_{0}-y_{1}\right\|\right| \text {. }
$$

Теорема доказана.

\section{4. Обсуждение итерационного процесса}

При использовании итерационного метода (14) для решения системы дифференциальных уравнений (4) с условиями (5)-(9) нам необходимо на каждом итерационном шаге решить две задачи: а) проинтегрировать «вперед» задачу Коши с отрицательно определенной матрицей $R$ и б) проинтегрировать «назад» задачу Коши с положительно определенной матрицей $-R$, т. е. нам необходимо решить уравнение вида

$$
x^{\prime}=R x+f_{n} ; \quad x(0)=0, \quad t \in[0, H] .
$$

Коэффициент жесткости ([7], с. 118) системы (26) определяется величиной $\mathrm{M} / \mathrm{m}$. Согласно лемме 1 , все собственные значения матрицы $R$ вещественны. Это позволяет использовать для решения задачи (26) любой $A(0)$ - устойчивый метод $\left(\left[{ }^{7}\right]\right.$, с. 119$)$.

Рассмотрим метод трапеций с переменным шагом

$$
\begin{gathered}
\left(I-0,5 h_{l+1} R\right) x^{l+1}=\left(I+0,5 h_{l+1} R\right) x^{l}+\int_{t_{l}}^{t_{l+1}} f_{n}(s) d s, \\
h_{l+1}=t_{l+1}-t_{l}, \quad l=1,2, \ldots, N .
\end{gathered}
$$

Этот метод наиболее точен среди неявных линейных многошаговых методов, обладающих свойством $A$-устойчивости $\left(\left[{ }^{7}\right]\right.$, с. 119$)$, и имеет второй порядок.

Те орем а 2. Eсли $g(\gamma)>0$, a также ${ }^{*} x^{l} \geqslant 0, \int_{t_{l}}^{t_{t+1}} f_{n}(s) d s \geqslant 0 u$

* 2-е и 3-е неравенства выполняются для каждой координаты. 


$$
h_{l+1} \leqslant 2 \min _{1 \leqslant i \leqslant n} \min _{1 \leqslant m \leqslant p} a_{i m} b_{i m}^{-1}\left[1-\lambda\left(4 \pi b_{i m}\right)^{-1} g_{i m i m}\right]^{-1},
$$

то решение $x^{l+1}$ системы (27) положительно.

Доказательств о. Условие $g(\gamma)>0$ гарантирует неразложимость матрицы $\left(I-0,5 h_{l+1} R\right)$. Используя свойство (9) и теорему 2 монографии $\left.{ }^{8}\right]$ (с. 353), получим, что матрица $\left(I-0,5 h_{l+1} R\right)^{-1}$ состоит из положительных элементов.

Рассмотрим матрицу $\left(I+h_{l+1} R\right)$. Недиагональные элементы этой матрицы положительны. Для этого чтобы и диагональные элементы были положительны, потребуем

$1+0,5 h_{l+1} a_{i m}^{-1} b_{i m}\left[\lambda\left(4 \pi b_{i m}\right)^{-1} g_{i m i m}-1\right] \geqslant 0, i=1,2, \ldots, n ; m=1,2, \ldots, p$.

Простой анализ последнего выражения приводит нас к неравенству (28).

Теорема доказана.

\section{5. Примеры метода дискретных ординат}

Пусть

$$
0=\mu_{0}<\mu_{1}<\ldots<\mu_{n}=1
$$

- некоторое разбиение отрезка $[0,1]$. Рассмотрим задачу (4)-(5) со следующими значениями параметров $a_{s m}, b_{s m}, g_{i l s m}$ :

$$
\begin{gathered}
a_{i m}=0,5 h_{\varphi}\left(\mu_{i}^{2}-\mu_{i-1}^{2}\right), \quad h_{\varphi}=2 \pi / p, \\
b_{i m}=h_{\varphi}\left(\mu_{i}-\mu_{i-1}\right), \quad \varphi_{m}=(m-0,5) h_{\varphi}, \\
g_{i l s m}=\int_{\mu_{t-1}}^{\mu_{t}} \int_{\varphi_{l-1}}^{\varphi_{t}} \int_{\mu_{s-1}}^{\mu_{s}} \int_{\varphi_{m-1}}^{\varphi_{m}} g(\gamma) d \mu d \varphi d \mu^{\prime} d \varphi^{\prime} .
\end{gathered}
$$

Для получения этих формул проинтегрируем уравнение (1) по переменным $\mu$ и $\varphi$ на множестве

$$
\Omega_{i m}=\left\{(\mu, \varphi) \mid \mu_{i-1} \leqslant \mu \leqslant \mu_{i}, \varphi_{m-1} \leqslant \varphi \leqslant \varphi_{m}\right\}
$$

при $\mu .>0$ и

$$
\Omega_{-i m}=\left\{(\mu, \varphi) \mid \mu_{-i+1} \leqslant \mu \leqslant \mu_{-i}, \varphi_{m-1} \leqslant \varphi \leqslant \varphi_{m}\right\}
$$

при $\mu<0$. Полагая, что функция $\mathfrak{J}(\tau, \mu, \varphi)$ постоянна по $\mu$ и $\varphi$ на множестве $\Omega_{\text {im }}$, получим метод $(29)-(32)$.

Можно показать, что матрицы $G_{i j}$, определенные в разделе 2 , являются циркулянтами ([ $\left.{ }^{1}\right]$, с. 6 ; $\left[{ }^{9}\right]$, с. 263$)$ с образующими векторами

при $p=2 q$ и

$$
\left(x_{i j}^{0} x_{i j}^{1} \ldots x_{i j}^{q-1} x_{i j}^{q} x_{i j}^{q-1} \ldots x_{i j}^{1}\right)
$$

$$
\left(x_{i j}^{0} x_{i j}^{1} \ldots x_{i j}^{q-1} x_{i j}^{q} x_{i j}^{q} x_{i j}^{q-1} \cdots x_{i j}^{1}\right)
$$

при $p=2 q+1$. Здесь

$$
x_{i j}^{l}=\int_{\mu_{t-1}}^{\mu_{t}} \int_{\varphi_{l}}^{\varphi_{t+1}} \int_{\mu_{f-1}}^{\mu_{f}} \int_{0}^{h_{\varphi}} g\left(\gamma^{\prime}\right) d \mu d \varphi d \mu^{\prime} d \varphi^{\prime},
$$




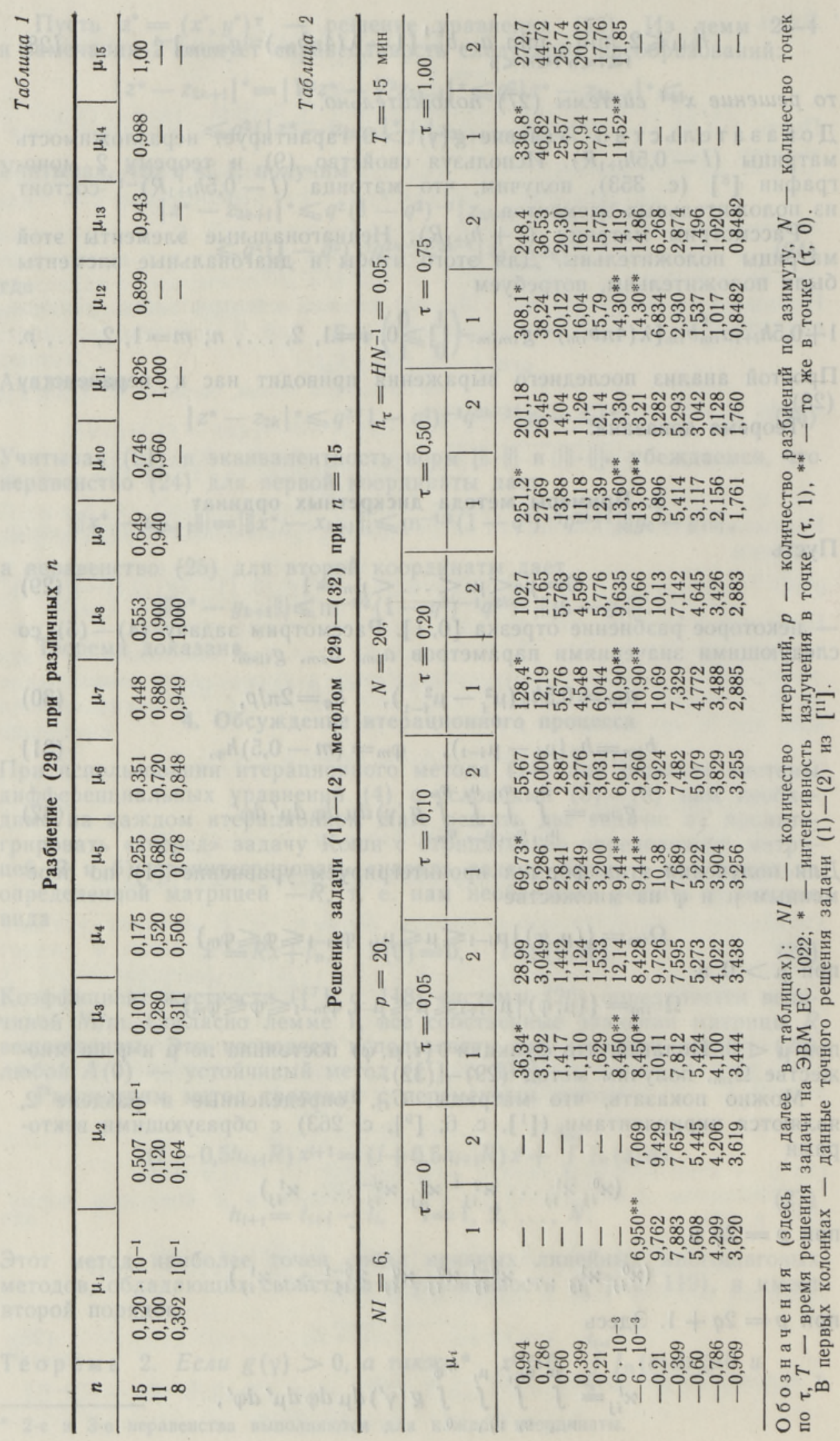




$$
\begin{gathered}
x_{-i j}^{l}=\int_{\mu_{-l}}^{\mu_{-l+1}} \int_{\varphi_{l}}^{\varphi_{l+1}} \int_{\mu_{l-1}}^{\mu_{f}} \int_{0}^{h_{\varphi}} g\left(\gamma^{\prime}\right) d \mu d \varphi d \mu^{\prime} d \varphi^{\prime}, \\
i, j=1,2, \ldots, n, \quad l=0,1,2, \ldots, q .
\end{gathered}
$$

Последнее позволяет с помощью унитарного преобразования (см. $\left[{ }^{1}\right]$ ) привести задачу (4)-(5) с параметрами (29)-(32) к удобному для алгоритмизации виду.

Рассмотрим еще один пример метода дискретных ординат из класса $(4)-(9)$. Положим

$$
h_{\mu}=1 / n, \mu_{j}=(j-0,5) h_{\mu}, h_{\varphi}=2 \pi / p, \varphi_{j}=(j-0,5) h_{\varphi},
$$

$g_{i l j m}=h_{\mu} h_{\varphi} g\left(\gamma_{i l j m}\right), \quad \cos \gamma_{i l j m}=\mu_{i} \mu_{j}+\sqrt{\left(1-\mu_{i}^{2}\right)\left(1-\mu_{j}^{2}\right)} \cos \left(\varphi_{m}-\varphi \iota\right)$,

$$
p_{i}=(2 n p)^{-1} \sum_{j=1}^{n} \sum_{l=1}^{p} g\left(\gamma_{i l j m}\right)
$$

$$
a_{i m}=\mu_{i} p_{i}, \quad b_{i m}=p_{i}, \quad u_{s m}=F g\left(\gamma_{s m}\right),
$$

$$
\cos \gamma_{s m}=\mu_{0} \mu_{s}+\sqrt{\left(1-\mu_{.0}^{2}\right)\left(1-\mu_{s}^{2}\right)} \cos \left(\varphi_{0}-\varphi_{s}\right) \text {. }
$$

Величину $p_{|s|} J_{s m}(\tau)$, где $J_{s m}(\tau)$ - решение задачи $(4)-(5)$ с параметрами (33)-(36), будем считать приближеңным решением $J\left(\tau, \mu_{s}, \varphi_{m}\right)$ задачи (1)-(2). Соотношения (33)-(36) можно получить, дискретизируя с учетом баланса частиц $\left(\left[{ }^{10}\right]\right.$, c. 127$)$ уравнение переноса (1)-(2) методом, предложенным в [ $\left.{ }^{1}\right]$.

\section{6. Численные результаты}

Задача (1)-(2) заменялась системой (4)-(5) с параметрами (29)(32). Система дифференциальных уравнений (4)-(5) с краевыми условиями (5) решалась итерационным методом (14). Для решения задачи Коши (26) использовался метод трапеций (27) с постоянным шагом $h_{\tau}=H / N(N$ из (27)).

В табл. 1 приведено разбиение (29) для $n=15,11,8$. Отметим, что при $n=15$ и 8 величина $0,5\left(\mu_{i-1}+\mu_{i}\right)$ есть узел квадратурной формулы Гаусса для отрезка $[0,1]$.

В табл. 2 приведено решение уравнения переноса (1)-(2) с начальными данными $\lambda=1, H=1, \mu_{0}=1, F=0,0025 \lambda$ и индикатрисой рассеяния из [11]. Расчеты проводились на ЭВМ ЕС 1022. Для срав-

Таблица 3

Погрешности решения задачи (1)-(2) при $n=15, \%$

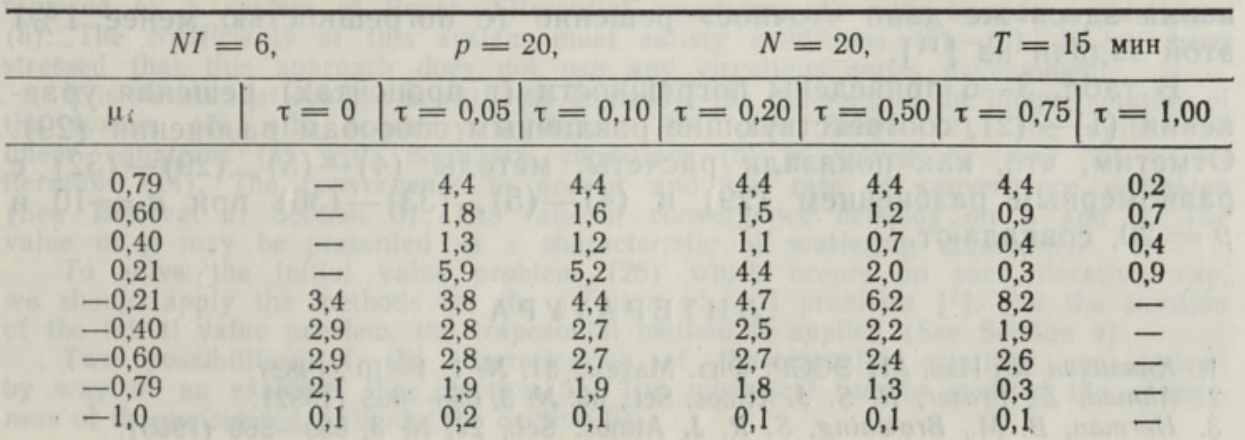


Погрешности решения задачи (1)-(2) при $n=11, \%$

\begin{tabular}{|c|c|c|c|c|c|c|c|c|}
\hline & \multicolumn{2}{|r|}{$N I=6$} & \multicolumn{2}{|c|}{$p=20$} & \multicolumn{2}{|c|}{$N=20$} & \multicolumn{2}{|c|}{$T=11$ мин } \\
\hline & $\mu_{i}$ & $\tau=0$ & $\tau=0,05$ & $\tau=0,10$ & $\tau=0,20$ & $\tau=0,50$ & $\tau=0,75$ & $\tau=1,00$ \\
\hline in & $\begin{array}{r}0,8 \\
0,6 \\
0,4 \\
0,2 \\
-0,2 \\
-0,4 \\
-0,6 \\
-0,8 \\
-0,98\end{array}$ & $\begin{array}{l}\bar{Z} \\
\overline{-} \\
\overline{4} \\
3,5 \\
3,3 \\
2,1 \\
3,0 \\
3,4\end{array}$ & $\begin{array}{l}6,7 \\
1,7 \\
2,3 \\
1,7 \\
4,3 \\
3,1 \\
1,9 \\
2,8 \\
3,1\end{array}$ & $\begin{array}{l}6,6 \\
1,8 \\
1,4 \\
1,5 \\
4,5 \\
3,0 \\
1,8 \\
2,8 \\
3,4\end{array}$ & $\begin{array}{l}6,2 \\
1,9 \\
1,5 \\
1,5 \\
4,0 \\
2,7 \\
1,7 \\
2,8 \\
3,4\end{array}$ & $\begin{array}{l}5,1 \\
2,0 \\
1,5 \\
1,2 \\
3,0 \\
1,9 \\
1,3 \\
2,6 \\
3,5\end{array}$ & $\begin{array}{l}4,3 \\
2,0 \\
1,5 \\
0,4 \\
1,7 \\
1,1 \\
1,6 \\
1,2 \\
3,6\end{array}$ & $\begin{array}{l}3,6 \\
1,8 \\
1,6 \\
0,2 \\
- \\
- \\
- \\
-\end{array}$ \\
\hline
\end{tabular}

Таблица 5

Погрешности решения задачи (1)-(2) при $n=10$ и равномерном разбиении (29), \%

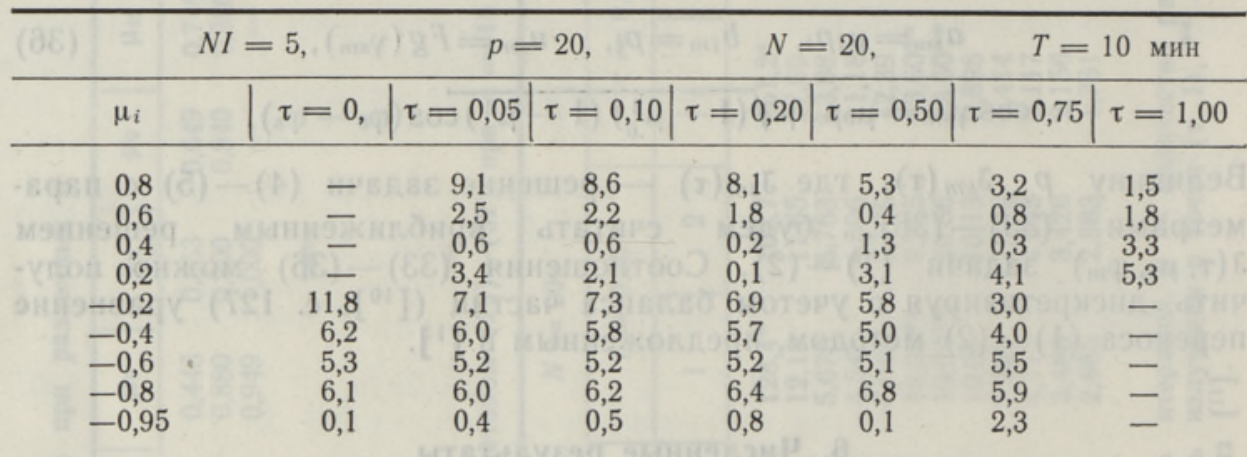

Таблица 6

Погрешности решения задачи (1)-(2) при $n=8, \%$

\begin{tabular}{c|c|c|c|c|c|c|c}
\hline \multicolumn{3}{c}{$N I=5}$, & \multicolumn{2}{c}{$p=20}$, & \multicolumn{2}{c}{$N=20}$, & \multicolumn{2}{c}{$T=7$ мин } \\
\hline$\mu_{i}$ & $\tau=0$ & $\tau=0,05$ & $\tau=0,10$ & $\tau=0,20$ & $\tau=0,50$ & $\tau=0,75$ & $\tau=1,00$ \\
\hline 0,8 & - & 20 & 20 & 19,6 & 17,8 & 16,7 & 15,8 \\
0,6 & - & 0,14 & 0,1 & 14,9 & 0,2 & 0,5 & 0,9 \\
0,4 & - & 0,4 & 1,2 & 1,1 & 0,9 & 0,7 & 0,7 \\
0,2 & - & 12,8 & 11,6 & 9,6 & 4,4 & 1,1 & 1,5 \\
$-0,20$ & 6,2 & 7,1 & 8,1 & 9,1 & 13,3 & 18,2 & - \\
$-0,41$ & 5,3 & 5,3 & 5,3 & 5,3 & 5,3 & 5,6 & - \\
$-0,6$ & 1,8 & 1,6 & 1,5 & 1,2 & 0,7 & 0,9 & - \\
$-0,76$ & 0,5 & 0,3 & 0,3 & 0,7 & 1,3 & 2,8 & - \\
$-1,00$ & 2,2 & 2,5 & 2,4 & 2,6 & 3,0 & 3,3 & -
\end{tabular}

нения здесь же дано «точное» решение (с погрешностью менее 1\%) этой задачи из [11].

В табл. 3-6 приведены погрешности (в процентах) решения уравнения (1)-(2), соответствующие различным способам разбиения (29). Отметим, что, как показали расчеты, методы (4)-(5), (29)-(32) с равномерным разбиением (29) и (4)-(5), (33)-(36) при $n=10$ и $p=20$, совпадают.

\section{Л И ТЕРАТ У Р А}

1. Князихин Ю. Изв. АН ЭССР, Физ. Матем., 31, № 1, 1-10 (1982).

2. Ahmad, Z., Fraser, R. S. J. Átmos. Sci., 39, № 3, 656-665 (1982).

3. Herman, B. M., Browning, S. R. J. Atmos. Sci., 22, № 3, 559-566 (1965). 
4. Ëschelbach, G. J. Quant. Ŝpectrosc. and Radiat. Transfer, i1, № 6, 757-765 (1971).

5. Рисс Ф., Сёкефальви-Надь Б. Лекции по функциональному анализу. М., «Мир», 1979.

6. Красносельский М. А., Вайникко Г. М., Забрейко П. П., Рутицкий Я. Б., Стеценко В. Я. Приближенное решение операторных уравнений. М., «Наука», 1969.

7. Современные численные методы решения обыкновенных дифференциальных уравнений (под ред. Дж. Холла, Дж. Уатта). М., «Мир», 1979.

8. Коллатц $A$. Функциональный анализ и вычислительная математика. М., «Мир», 1969.

9. Беллман $P$. Введение в теорию матриц. М., «Наука», 1976.

10. Смелов В. В. Лекции по теории переноса нейтронов. М., Атомиздат, 1978.

11. Standard Procedures to Compute Atmospheric Radiative Transfer in a Scattering Atmosphere (ed. by Jac. Lenoble). IAMAP, Radiation Commission, Boulder, USA, July, 1977.

\section{Институт астрофизики и физики атмосферы} Академии наук Эстонской ССР
Поступила в редакцию

29 апреля 1982

После переработки 10 октября 1982

\section{J. KNJAZIHHIN}

\section{DISKREETSETE ORDINAATIDE MEETODI KASUTATAVUS KIIRGUSLEVI VORRANDI LAHENDAMISEKS HOMOGEENSE, TASAPARALLEELSE, ANISOTROOPSELT HAJUTAVA ATMOSFÄĂRI KORRAL. 2}

Artiklis on jätkatud diskreetsete ordinaatide meetodi käsitlemist selle vahetul rakendamisel, kasutamata hajumisindikatrissi reaksarendusi Legendre'i polünoomide kaudu. Ơlesanne taandub harilike diferentsiaalvôrrandite süsteemi rajaülesandele. Peatähelepanu on koondatud rajaülesande lahendamisele iteratsioonimeetodil. On näidatud koonduvus ja geomeetriline koonduvuskiirus. On käsitletud ka vastava Cauchy ülesande lahendamist (Cauchy ülesanne on iteratsioonimeetodi sammuks), arvestades diferentsiaalvõrrandite süsteemi jäikust.

\section{J. KNJAZIHHIN}

\section{INVESTIGATION OF THE DISCRETE ORDINATES SOLUTION OF THE RADIATIVE TRANSFER EQUATION IN THE HOMOGENEOUS, PLANE-PARALLEL, ANISOTROPICALLY SCATTERING ATMOSPHERE. 2}

The present paper presents the second part of the investigation of the discrete ordinates solution of the radiative transfer equation in the homogeneous, plane-parallel, anisotropically scattering atmosphere. The following variant of this method is considered: the integro-differential equation (1) with boundary conditions (2) is replaced by a system of linear differential equations (4) with boundary conditions (5). The coefficients of this system must satisfy conditions (6)-(9). It has been stressed that this approach does not use any circuitous series developments.

It is shown that conditions (6) - (9) guarantee the existence of the unique solution of the system (4) with boundary conditions (5) (See Section 2). The system of linear equations (4) with boundary conditions (5) is solved by way of Seidel iteration (14). The convergence is proved and the rate of convergence estimated (See Theorem 1, Section 3). The rate of convergence depends on $\lambda$ and $g$. The value of $g$ may be presented as a characteristic of scattering anisotropy.

To solve the initial value problem (26) which occurs on each iterative step, we should apply the methods for the solution of stiff problems $\left[{ }^{7}\right]$. For the solution of the initial value problem, the trapezoidal method is applied (See Section 4).

Two possibilities of the discretization of the transfer equation are derived by way of an example (See Section 5). The numerical results confirm the correctness of the considered method (See Section 6). 